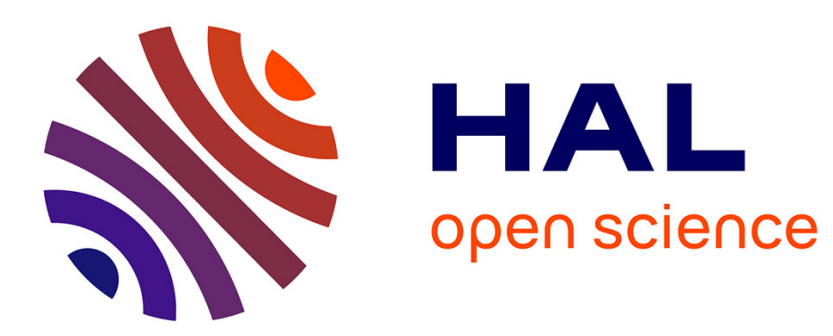

\title{
Qualité des viandes de volaille: le rôle des matières grasses alimentaires
}

\author{
M. Lessire
}

\section{To cite this version:}

M. Lessire. Qualité des viandes de volaille: le rôle des matières grasses alimentaires. Productions Animales, 1995, 8 (5), pp.335-340. hal-00896130

\section{HAL Id: hal-00896130 https://hal.science/hal-00896130}

Submitted on 1 Jan 1995

HAL is a multi-disciplinary open access archive for the deposit and dissemination of scientific research documents, whether they are published or not. The documents may come from teaching and research institutions in France or abroad, or from public or private research centers.
L'archive ouverte pluridisciplinaire HAL, est destinée au dépôt et à la diffusion de documents scientifiques de niveau recherche, publiés ou non, émanant des établissements d'enseignement et de recherche français ou étrangers, des laboratoires publics ou privés. 
INRA Prod. Anim., $1995,8(5), 335-340$

\section{LESSIRE}

INRA Station de Recherches Avicoles 37380 Nouzilly

\title{
Qualité des viandes de volaille : le rôle des matières grasses alimentaires
}

\author{
La qualité des viandes et des produits animaux est devenue une \\ préoccupation majeure pour tous les partenaires de la filière avicole. \\ La teneur en lipides des viandes, critère de qualité pour le \\ consommateur, dépend des caractéristiques nutritionnelles des \\ aliments ingérés et des quantité et qualité des matières grasses \\ ajoutées.
}

L'éleveur, le producteur avicole, ont pour objectif de produire au moindre coût une quantité importante d'animaux : poulets, dindons... Ces coûts de productions dépendent largement du prix de l'aliment et surtout des ingrédients énergétiques qui le composent. Le niveau énergétique défini par le formulateur revêt donc une importance considérable non seulement pour le profit du producteur (Pesti 1994) mais aussi pour le choix des matières premières constitutives des rations. Ainsi l'incorporation de matières grasses dont la teneur en énergie atteint le double, voire le triple de celle des céréales et tourteaux est particulièrement justifiée dans les aliments concentrés modernes.

Commercialiser la plus grande proportion de l'animal est l'objectif de l'abattoir, aussi toute perte de rendement est préjudiciable. Les excès de graisse abdominale et viscérale éliminés lors de la préparation et de la découpe (environ $35 \%$ et $90 \%$ des poulets et des dindes produits en France ont été découpés en 1994) seront autant de pertes que l'abattoir devra répercuter. En outre ces graisses excessives accroissent les coûts de nettoyage des chaînes d'abattage et de dépollution des effluents de l'abattoir.

Enfin le consommateur, qui apprécie de moins en moins les aliments gras, est également sensible aux dépôts adipeux qu'il élimine lui-même ou qui fondent lors de la cuisson, or la masse de ces dépôts s'est largement accrue depuis 30 ans (Leclercq 1989).

Abattoirs et consommateurs sont donc concernés par l'état d'engraissement des carcasses des volailles. Quel rôle jouent les matières grasses alimentaires dans les quantités et qualités des dépôts adipeux des oiseaux? Leur nature peut-elle modifier la qualité organoleptique et diététique des viandes de volaille?

\section{1 / Apport énergétique de la ration et état d'engraissement}

Les dépôts corporels de lipides varient chez les oiseaux en fonction de nombreux paramètres ; les plus connus sont d'origine génétique et nutritionnelle (Fisher 1984). L'espèce, l'âge et le sexe de l'oiseau sont en effet susceptibles de modifier l'état d'engraissement. Ainsi, à l'abattage (Larbier et Leclercq 1992), le poulet renferme en moyenne plus de 
lipides que le dindonneau : 17,2 vs $8,4 \%$ et les femelles sont plus grasses que les mâles : 18,9 vs $15,5 \%$ et 9,7 vs $7,1 \%$ chez le poulet et le dindonneau respectivement. Ces différences d'engraissement existent pratiquement dès la naissance chez le poulet et n'apparaissent qu'à l'âge de 84 jours chez la dinde (figure 1). L'état d'engraissement du canard est très proche de celui du poulet. Il en est de même de la proportion de gras abdominal : $2,9 \%$ chez le canard (Leclercq et de Carville 1986), 2,7\% chez le poulet mâle et seulement $0,6 \%$ chez le dindonneau (Kouba et al 1992). Ce dépôt, éliminé à l'éviscération, a constitué le critère privilégié de sélection de lignées de poulets maigres puisqu'il est corrélé aux lipides totaux de la carcasse (Delpech et Ricard 1965).

L'apport de matières grasses : huiles, graisses animales ou graines oléagineuses, dans les rations a permis d'en accroître la densité énergétique améliorant ainsi les performances de croissance des oiseaux. Cependant, tout accroissement de l'ingéré énergétique s'accompagne d'un dépôt accru de lipides corporels (Leclercq 1986). Dans de telles conditions, il conviendrait de séparer l'effet spécifique des matières grasses de celui d'un accroissement de la densité énergétique de la ration, et donc de raisonner (ou formuler) les aliments à rapport protéines sur calories (P/C) constant. Selon Combs (1962), la corrélation négative entre le rapport $\mathrm{P} / \mathrm{C}$ et la teneur en lipides corporels montre que le poulet est capable d'augmenter son ingéré total pour satisfaire son besoin en protéines. Cette hypothèse a été reprise par de nombreux auteurs (voir revue de Fisher 1984).
A l'inverse, la réduction des lipides corporels par des aliments riches en protéines, et donc à rapports $\mathrm{P} / \mathrm{C}$ plus élevés, s'expliquerait par le coût énergétique élevé de l'élimination sous forme d'acide urique de l'azote excédentaire par rapport aux besoins. Cependant, des aliments renfermant des protéines peu digestibles telles que la farine de plume, et qui ne conduisent donc pas à une excrétion accrue d'acide urique réduisent de la même façon les dépôts gras (Griffiths et al 1977). Il en est de même de certains acides aminés tels que l'acide glutamique (Velu et al 1972). Ces résultats qui sont illustrés à la figure 2 , montrent que la réponse des poulets à une élévation du rapport protéines sur calories est curvilinéaire, le minimum de lipides totaux déposés est de $80 \mathrm{~g} / \mathrm{kg}$ de poids vif, le maximum atteint $180 \mathrm{~g} / \mathrm{kg}$. Un tel schéma permet donc de prévoir la masse lipidique des oiseaux en fonction du rapport $\mathrm{P} / \mathrm{C}$ de l'aliment.

Dans de telles conditions, modifier le niveau énergétique de la ration par un apport de glucides ou de lipides à teneur constante en protéines aura un effet sur l'engraissement que l'on pourra estimer à partir du calcul des rapports $\mathrm{P} / \mathrm{E}$ (Fisher 1984). A l'inverse, modifier l'énergie métabolisable d'un aliment à rapport $\mathrm{P} / \mathrm{E}$ constant aura des conséquences complexes tant sur la formule alimentaire que sur la réponse des oiseaux.

Il est classiquement admis que la substitution des glucides par des lipides diminue la synthèse hépatique d'acides gras. Cette modification est attribuée plus à une réduction de l'apport de glucides qu'à l'apparition de lipides dans l'aliment (Hilliard et al 1980).

Figure 1. Effets de l'âge et du sexe sur la teneur en lipides corporels des différentes espèces aviaires (Larbier et Leclercq 1992).

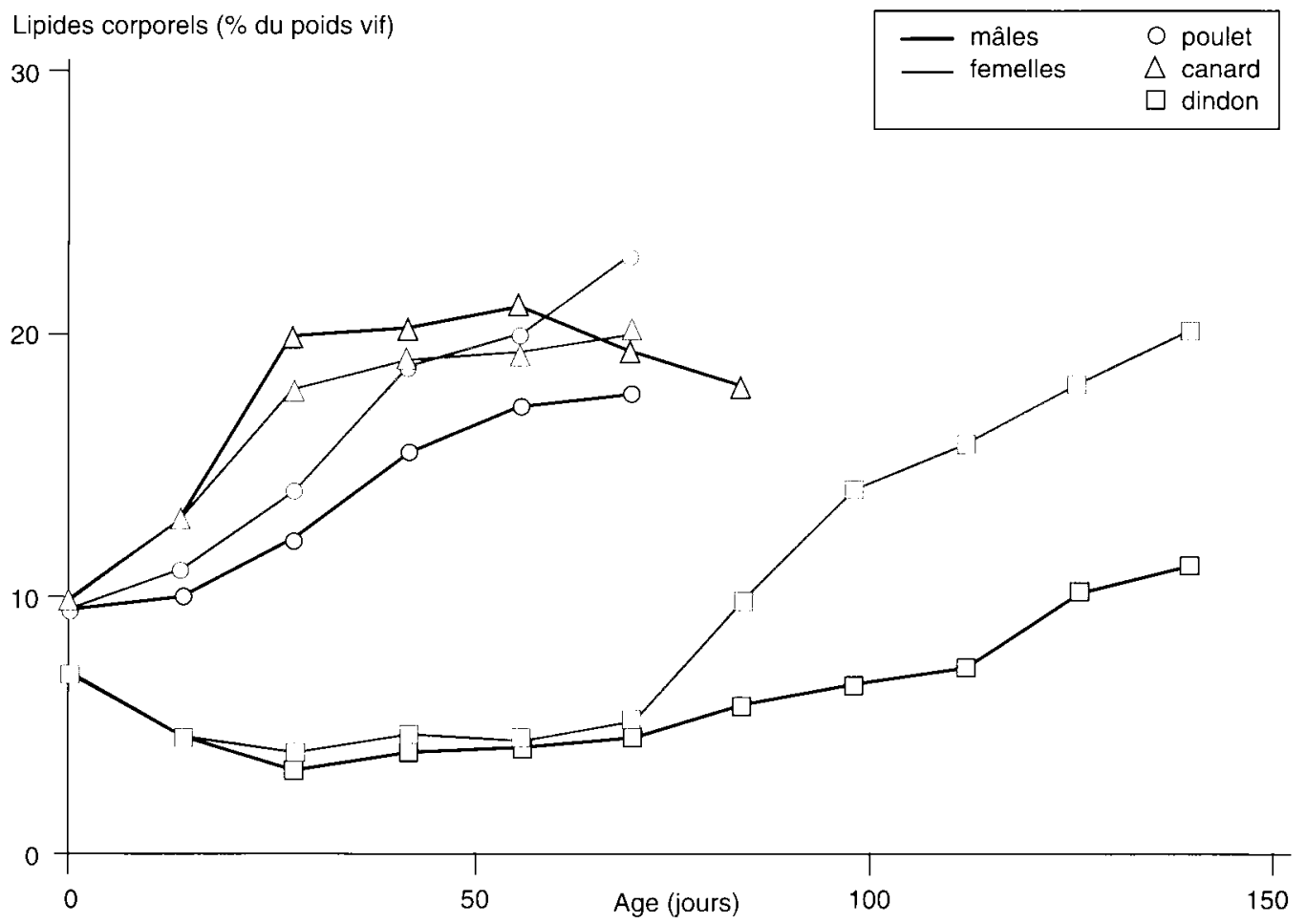


Une réduction de la synthèse d'acides gras devrait donc conduire à des dépôts réduits. Or, à quantité égale d'aliment ingéré, la croissance du poulet et la rétention d'énergie augmentent avec des aliments dont on a substitué une partie des glucides par des lipides (substitution isoénergétique), mais sa composition corporelle n'est pas modifiée (Grimbergen et al 1982). La diminution de la synthèse hépatique pourrait être compensée par le dépôt direct des acides gras absorbés.

Cependant, de tels essais supposent une substitution isoénergétique parfaite entre ingrédients alimentaires, qu'ils soient d'origine glucidique ou lipidique. Or, de nombreux facteurs et interactions sont susceptibles de faire varier les valeurs d'énergie métabolisable des lipides alimentaires (Wiseman 1984), conduisant ainsi à des substitutions qui ne sont pas isoénergétiques et donc à des aliments n'ayant pas les mêmes rapports protéines sur calories. Ce biais méthodologique peut être à l'origine des nombreuses divergences observées dans la bibliographie et la mesure in vivo systématique de l'énergie métabolisable, ou mieux de l'énergie nette des aliments expérimentaux devrait être effectuée parallèlement aux mesures de composition corporelle.

\section{2 / Acides gras alimentaires et qualité des carcasses}

La composition en acides gras des lipides des tissus dépend largement de l'importance relative de la lipogénèse hépatique et du dépôt direct des acides gras ingérés. Les dépots adipeux de poulets recevant un aliment dépourvu de lipides (tableau 1) se composent en majorité d'acide palmitique $(25 \%)$ et d'acide oléique (45 à $58 \%$ ). Pour ce dernier acide, la valeur la plus élevée est observée sur des animaux dont la ration est totalement carencée en lipides (Bottino et al 1970) et dont le taux de survie moyen est faible, de l'ordre de $50 \%$. Dans l'autre cas, il s'agit de poulets ne recevant pas de lipides exogènes mais l'aliment distribué renfermait une pro-

Figure 2. Influence du rapport protéines sur énergie de l'aliment sur les quantités de lipides déposés dans la carcasse de poulets mâles, femelles ou des 2 sexes (synthèse de différents essais, Fisher 1984).

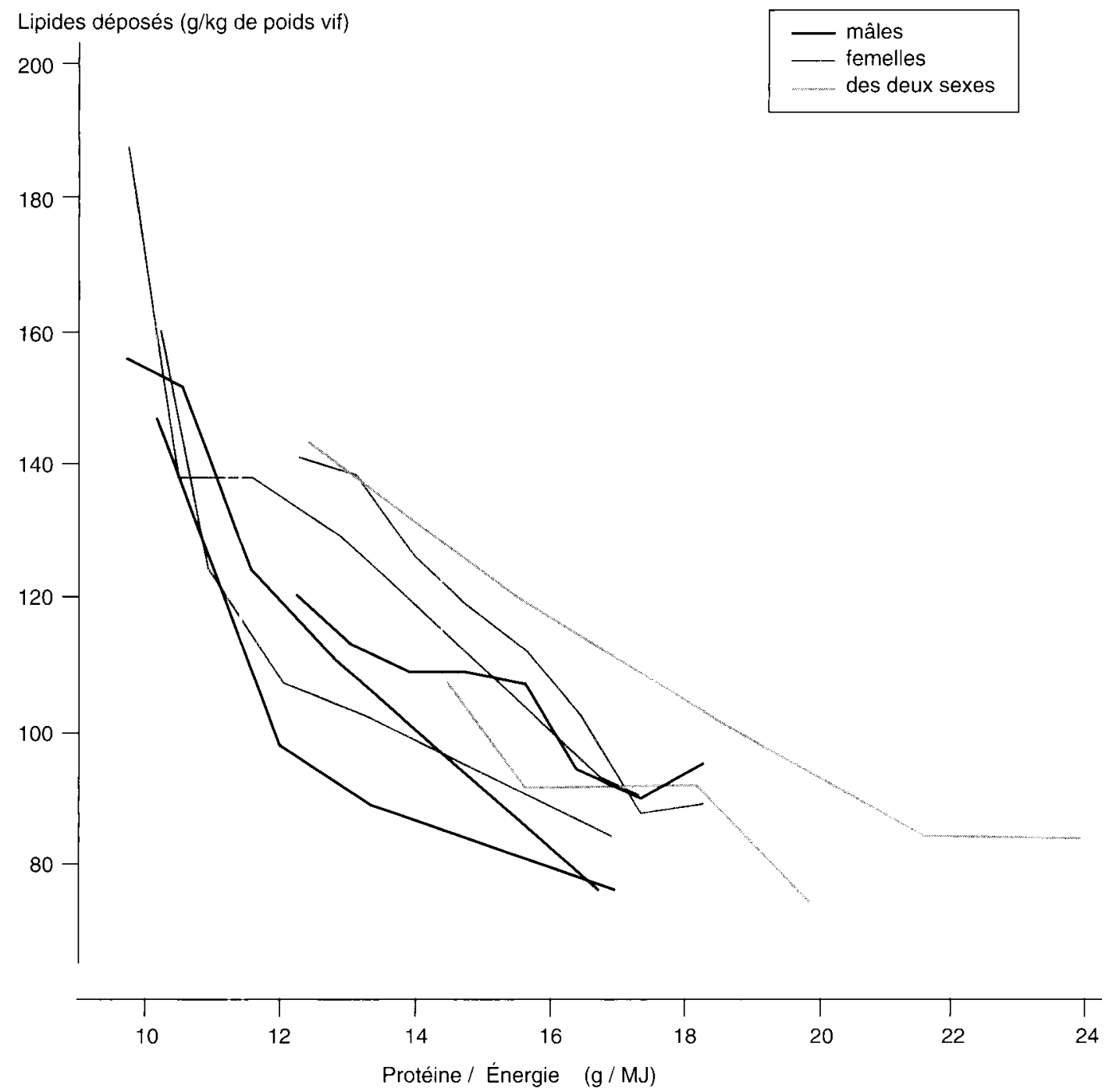

A même apport protéique, plus la densité énergétique de l'aliment est élevée et plus les dépôts lipidiques augmentent. 
Tableau 1. Composition en acides gras (\%) des tissus adipeux de poulets ne recevant pas de lipides dans leur alimentation.

\begin{tabular}{|l|c|c|c|}
\hline $\begin{array}{l}\text { Acides gras } \\
\text { des dépôts }\end{array}$ & $\begin{array}{c}\text { Mélanges de } \\
\text { tissus adipeux }^{(1)}\end{array}$ & Gras abdominal $^{(2)}$ & Gras sous-cutané $^{(2)}$ \\
\hline C $14: 0$ & 0,9 & 0,46 & 0,48 \\
C $16: 1$ & 25,1 & 25,04 & 25,02 \\
C $16: 0$ & 6,4 & 7,26 & 7,16 \\
C $18: 0$ & 8,4 & 6,54 & 6,42 \\
C $18: 1$ & 58,1 & 46,15 & 45,28 \\
C $18: 2$ & & 12,62 & 13,00 \\
C $18: 3$ & & 0,41 & 0,46 \\
\hline
\end{tabular}

(1) Bottino et al 1970

(2) Blanch et al 1993
D'une façon générale, le profil en acides gras des dépôts reflète la composition des lipides ingérés. Des corrélations significatives entre la nature des lipides alimentaires et celle des dépôts adipeux ont été mises en évidence, en particulier pour les acides gras insaturés (Pinchasov et Nir 1992, Gaudron et al 1993, Scaife et al 1994).

En outre il est à noter que les différentes sources de matières grasses utilisées dans ces essais n'ont eu aucun effet sur l'engraissement des oiseaux, qui était estimé par la proportion de gras abdominal et/ou la teneur en matières grasses des cuisses et pilons.

Ces changements de profils en acides gras des dépôts adipeux du poulet s'accompagnent d'une modification de l'aspect des carcasses de poulets qui ont été notées plus fermes et sèches au toucher dans le cas d'animaux dont les aliments sont supplémentés avec du suif (Edwards et al 1973, Gaudron et al 1993). A l'inverse, des carcasses de poulets nourris avec des huiles insaturées ont des graisses plus molles; l'appréciation de la qualité de la carcasse peut donc dépendre de la teneur en acides gras saturés (Gaudron et al 1993) des lipides déposés et donc ingérés par l'oiseau. Afin obtenir des carcasses considérées comme de bonne qualité visuelle, ces auteurs ont préconisé des rations dont la fraction lipidique serait constituée de $20 \%$ d'acide palmitique et de $24 \%$ d'acide linoléique.

En dehors de l'aspect de la carcasse, la saveur peut également être altérée par l'utilisation de différentes sources de lipides alimentaires. Il en est ainsi des lipides riches en acides gras polyinsaturés, très susceptibles à l'oxydation, ou de lipides déjà oxydés avant d'être consommés par les oiseaux. Ainsi une incorporation de farine ou d'huile de poisson peut provoquer l'apparition d'un goût désagréable que l'on attribue à l'oxydation des acides gras insaturés à longue chaîne (série n-3). Cette altération est rencontrée principa-

Tableau 2. Composition en acides gras (\%) des dépôts lipidiques du poulet en fonction de la nature des lipides ingérés.

\begin{tabular}{|c|c|c|c|c|c|c|c|c|c|}
\hline $\begin{array}{l}\text { Acides gras } \\
\text { des dépôts }(\%)\end{array}$ & Suif & $\underset{12,}{\text { Saindoux }}$ & $\begin{array}{l}\text { Huile de } \\
\text { palme }\end{array}$ & $\begin{array}{l}\text { Huile de } \\
\text { maïs }\end{array}$ & $\begin{array}{l}\text { Huile de } \\
\text { soja }\end{array}$ & $\begin{array}{l}\text { Graisse de } \\
\text { volaille }\end{array}$ & $\begin{array}{l}\text { Huile de } \\
\qquad \operatorname{lin}\end{array}$ & $\begin{array}{l}\text { Huile de } \\
\text { poisson }\end{array}$ & $\begin{array}{l}\text { Huile de } \\
\text { hareng }{ }^{(2)}\end{array}$ \\
\hline $14: 0$ & 2,1 & 1,2 & 0,6 & 0,2 & 0,6 & 1,0 & 0,2 & 2,7 & 6,4 \\
\hline $16: 0$ & 21,4 & 25,7 & 27,4 & 12,0 & 17,9 & 21,9 & 8,3 & 21,4 & 24,2 \\
\hline $16: 1$ & 6,2 & 4,0 & 5,4 & 1,0 & 3,2 & 7,1 & 1,0 & 4,2 & 11,7 \\
\hline $18: 0$ & 9,8 & 9,7 & 4,8 & 3,7 & 4,9 & 7,4 & 7,5 & 5,8 & 7,4 \\
\hline $18: 1$ & 38,6 & 43,8 & 46,2 & 30,3 & 29,2 & 35,4 & 31,8 & 28,0 & 29,5 \\
\hline $18: 2$ & 19,6 & 14,2 & 12,9 & 51,9 & 28,0 & 24,9 & 16,9 & 9,5 & 8,0 \\
\hline $18: 3$ & 1,0 & 0,9 & 0,5 & 1,4 & 3,8 & 0,9 & 33,8 & 1,5 & 4,8 \\
\hline $20: 0$ & & 0,0 & & & 0,1 & & & 0,5 & 1,3 \\
\hline $20: 1$ & & & & & 0,5 & & & 3,5 & \\
\hline $20: 4$ & 0,7 & & & & 0,3 & 1,1 & & 0,3 & \\
\hline $20: 5$ & & & & & 0,3 & & & 2,4 & \\
\hline $20: 6$ & & & & & & & & & 1,8 \\
\hline $22: 5$ & & & & & & & & 1,0 & \\
\hline $22: 6$ & & & & & 0,2 & & & 2,8 & \\
\hline
\end{tabular}


lement dans des viandes cuites avant stockage (Poste 1990) alors qu'aucun goût de poisson n'est détecté sur une viande analysée juste après la cuisson (Huang et al 1990, Miller et Huang 1993). De nombreux auteurs (voir revue de Sheeby et al 1993) mentionnent l'intérêt de larges supplémentations en vitamine $\mathrm{E}$ (160 UI/kg) non seulement sur les performances zootechniques : mortalité, croissance, efficacité alimentaire, mais aussi sur la non altération des qualités organoleptiques du poulet pendant le stockage. Ainsi, une viande fraîche ou congelée et dégustée aussitôt après cuisson est parfaitement acceptée quelles que soient la nature des lipides ingérés par l'oiseau et la teneur en vitamine $\mathrm{E}$ de l'aliment. A l'inverse, la supplémentation de l'aliment en cette vitamine contribue au maintien des qualités organoleptiques du poulet si la dégustation est effectuée après une conservation de 3 jours $\left(+4^{\circ} \mathrm{C}\right)$ de la viande cuite, cet effet est surtout important pour une viande préalablement congelée. La vitamine E (200 mg d' $\alpha$-tocophérol/kg d'aliment) distribuée pendant les 3 à 4 semaines qui précèdent l'abattage, sature les tissus gras des poulets, améliorant ainsi leur résistance à l'oxydation (Brandon et al 1993) et leur acceptabilité (Miller and Huang 1993).

\section{Conclusion}

Il ne fait aucun doute que le poulet industriel actuel diffère de ses ancêtres récents éle- vés il y a seulement quelques décennies. Il est abattu plus jeune et sa carcasse est plus grasse. Cette caractéristique que certains qualifieront de non-qualité s'est développée chez cet animal dont la propension à déposer les lipides ingérés est élevée. Les matières grasses incorporées dans l'aliment ont vraisemblablement contribué à cet engraissement, mais sans doute moins par leur rôle direct que par les modifications techniques qu'elles ont apportées aux fabricants d'aliments composés, en particulier en leur permettant d'accroître la concentration énergétique des rations.

Malgré cela, la viande de volaille reste encore maigre par rapport à d'autres produits carnés dont la consommation décroît. En outre, les lipides contenus sont riches en acide gras insaturés ce qui conduit encore plus les diététiciens à en recommander la consommation. Enfin la propension qu'ont les oiseaux à incorporer dans leurs tissus les acides gras alimentaires y compris les acides polyinsaturés à longue chaîne devrait conduire le fabricant d'aliment à utiliser des matières premières riches en ces acides afin de suivre les recommandations du corps médical pour qui ces acides gras diminuent le taux de cholestérol circulant, limitant ainsi les risques cardio-vasculaires chez l'homme. Cependant une telle pratique peut avoir des répercussions sur l'oxydation des produits et donc sur leur acceptabilité, leur "qualité », chez le consommateur.

\section{Références bibliographiques}

Blanch A., Barrota A.C., Puchal F., 1993. Effects of dietary linseed oil and palm oil on fatty acid profile of carcass fat depots in broiler chickens. In : Quality of poultry meat. WPSA, Tours (France), 1993/10/04$08,86-92$

Bottino N.R., Anderson R.E., Reiser R., 1970. Animal endogenous triglycerides : 2. Rat and chicken adipose tissue. Lipids, 5, 165-170.

Brandon S., Morrissey P.A., Buckley D.J., Grigg M, 1993. Influence of dietary $\alpha$-tocopheryl acetate on the oxidative stability of chicken tissues. In : Quality of poultry meat. WPSA, Tours (France), 1993/10/04-08, 397-403.

Combs G.F., 1962. The interrelationships of dietary energy and protein in poultry nutrition. In : J.T. Morgan and D. Lewis (eds), Nutrition of Pigs and Poultry, 127-147. Butterworths, London (UK).

Delpech P., Ricard F.H., 1965. Relation entre les dépôts adipeux et les lipides corporels chez le poulet. Ann. Zootech., 14, 181-189.

Edwards H.M.Jr, Hart P., 1971. Carcass composition of chickens fed carbohydrate-free diets containing various lipid energy sources. J. Nutr., 101, 989-996.

Edwards H.M.Jr, Denman F., Abou-Ashour A, Nugara D., 1973. Carcass composition studies. 1.
Influence of age, sex and type of dietary fat supplementation on total carcass and fatty composition. Poultry Sci., 52, 934-948.

Fisher C., 1984. Fat deposition in broilers. In :Wiseman J. (ed), Fats in Animal Nutrition, 437-470. Butterworth, Nottingham (UK).

Gaudron I., Castaing J., Magnin M., Lessire M., Barrier-Guillot B., Bureau J., Zwick J.L., Messager B., 1993. Influence de l'incorporation de différentes matières grasses dans l'aliment sur la qualité des carcasses de poulet de chair. In : Quality of poultry meat. WPSA, Tours (France), 1993/10/04-08, 93-102.

Griffiths L., Leeson S., Summers J.D., 1977. Fat deposition in broilers: effect of dietary energy to protein balance, and early life caloric restriction on productive performance and abdominal fat pad size. Poultry Sci., 56, 538-546.

Grimbergen A.H.M., Stappers H.P., Cornelissen J.P., 1982. The influence of an isocaloric substitution of soyabean oil for carbohydrates and of the nutrient density of the feed on growth and efficiency of energy utilization in broiler chickens. Neth. J. Agric. Sci., 30, 115-125.

Hilliard B.L., Lundin P., Clarke S.D., 1980. Essentiality of dietary carbohydrate for maintenance of liver lipogenesis in the chick. J. Nutr., 110, 1533-1542.

\section{L'ajout de matières grasses à l'aliment peut aussi modifier l'aspect de la carcasse et altérer la saveur de la viande.}


Huang Z.B., Leibovitz H., Lee C.M., Millar R., 1990. Effect of dietary fish oil on $\omega-3$ fatty acid levels in chickens eggs and thigh flesh. J. Agric. Food Chem., $38,743-747$.

Kouba N., Catheline D., Leclercq B., 1992. Lipogenesis in turkeys and chickens : A study of body composition and liver lipogenic enzyme activities. Brit. Poult. Sci., 3, 1003-1014.

Larbier M., Leclercq B., 1992. Nutrition et alimentation des volailles. INRA Editions, Versailles (France), $352 \mathrm{p}$.

Leclercq B., 1986. Energy requirements of avian species. In : Fisher C. and Boorman K.N (eds), Nutrient Requirements of Poultry and Nutritional Research, 125-141. Butterworths, London (UK).

Leclercq B., 1989. Possibilités d'obtention et intérêt de génotypes maigres en aviculture. INRA Prod. Anim., 2 (4), 275-286.

Leclercq B., de Carville H., 1986. Growth and body composition of muscovy ducks. In : Farrell D.J. and Stapleton P. (eds), Duck Production science and world practice, 102-109. University of New England, Armidale (Australia).

Leclercq B., Blum J.C., Jacquot R., 1965. Etude de l'utilisation de l'acide élaïdique au cours de l'ovogénèse de Gallus gallus L. : son incorporation dans les graisses de réserve et dans les lipides du vitellus de l'œuf pondu. C.R. Acad. Sci., Paris, t. 261, 3197-3200.

Miller E.L., Huang Y.X., 1993. Improving the nutritional value of broiler meat through increased $n-3$ fatty acid and Vitamin E content. In : Quality of poultry meat. WPSA, Tours (France), 1993/10/04-08, 404-411.
Pesti G.M., 1994. Description d'un modèle de formulation permettant un profit maximum. Conférence avicole WPSA - SIMAVIP, Villepinte (FRA), 1994/2/15. In : Nutrition et Alimentation Avicoles : vers une démarche intégrée. Cahier du SIMAVIP $\mathrm{n}^{\circ}$ 9, 25-46.

Pinchasov Y., Nir I., 1992. Effect of dietary polyunsaturated fatty acid concentration on performance, fat deposition and carcass fatty acid composition in broiler chickens. Poultry Sci., 71, 1504-1512.

Poste L.M., 1990. A sensory perception of effects of feeds on flavor in meats: Poultry meats. J. Anim. Sci., 68, 4414-4420.

Scaife J.R., Moyo J., Galbraith H., Mickie W., Campbell V., 1994. Effect of different dietary supplemental fats and oils on the tissue fatty acid composition and growth of female broilers. Brit. Poult. Sci., 35, 107-118.

Sheeby P.J.A., Morrissey P.A., Buckley D.J., Frigg M., 1993. Lipid oxydation and dietary vitamin E supplementation in broilers. In : quality of poultry meat. WPSA, Tours (France), 1993/10/04-08, 380388.

Velu J.G., Scott H.M., Baker D.H., 1972. Body composition and nutrient utilization of chicks fed amino acid diets containing graded amount of either isoleucine and lysine. J. Nutr., 102, 741-748.

Wiseman J., 1984. Assessment of the digestible and metabolizable energy of fats for non-ruminants. In : Wiseman J. (ed), Fats in Animal Nutrition, 277-297. Butterworth, Nottingham (UK).

\begin{abstract}
Poultry meat quality: the role of dietary fats.
Poultry meat consumption is increasing at the expense of other meat sources. Such an increase is based on the lower lipid content of poultry meat. Chickens however have been becoming fatter over the last years. The aim of this review is to analyze the dietary factors which are responsible of fat deposition in poultry.
\end{abstract}

Abstract

The increase of the dietary energy level and particularly of the calorie to protein ratio has more effect on fat deposition than the actual lipid intake level. Conversely, the origin and the composition of dietary lipids may modify the fatty acid profiles of the adipose tissues and thus change the organoleptic characteristics and the resultant nutritional value of the poultry meat.

LESSIRE M., 1995. Qualité des viandes de volaille : le rôle des matières grasses alimentaires. INRA Prod. Anim., 8 (5), 335-340. 